\title{
Sensitive X-ray Detectors Synthesised from $\mathrm{CsPbBr}_{3}$
}

\author{
Logan J. Forth, Student Member IEEE, Mingqing Wang, Issy Braddock, Jia C. Khong, Rob Moss, \\ Paul Sellin, Member IEEE, Kwang L. Choy and Robert Speller
}

\begin{abstract}
The materials used in detection of high energy photons are of primary importance in the construction of efficient, cost effective and sensitive detectors. Current research into Perovskites for solar cell technology has stimulated interest in their potential alternative uses, one of which is in direct photon conversion radiation detectors, owed primarily to their high-Z elemental composition twinned with exceptional charge carrier transport properties. Here, the Perovskite $\mathrm{CsPbBr}_{3}$ has been synthesised through solution growth. The raw $\mathrm{CsPbBr}_{3}$ was a granular powder which was formed into disks of 8 mm diameter and 1-2 $\mathrm{mm}$ thickness by two methods: 1). the powders were pressed into pellets using a hydraulic press or 2). sealed in a quartz ampoule under vacuum and then melted and quenched to form a polycrystalline solid which was cut to size. Metallic contacts were deposited on the front and back faces to permit charge collection. The results from the pressed devices are promising, particularly given that the production method is cost effective, repeatable and scalable. The solid-from-melt devices show similar performance but further development is required to optimise the production method.
\end{abstract}

Index Terms-Perovskite, X-ray Detection, Direct Detector, Crystal Growth, Device Fabrication

\section{INTRODUCTION}

Perovskite materials have seen a surge of interest after recent developments in the realm of photovoltaics [3]. Compared with previous materials their photo-electronic conversion efficiency has risen remarkably quickly relative to their competitors. This is due in no small part to their high $\mu \tau$-product [1], a major figure of merit in the field of solar cell research, $\mu$ relating to the free charge carrier mobility within the material and $\tau$ to the median lifetime of such charge carriers, the combination of which leads to high charge collection efficiency. A high $\mu \tau$-product is not just assistive to materials used for solar conversion, but of all photodetecting materials. Twinned with the case that these perovskite materials are primarily composed of heavy elements, Lead along with Bromine and Caesium, we have the design for an ideal $\mathrm{X}$ and gamma ray detector [1]. When compared with current X-ray detectors, Silicon, -Se,

Manuscript Received July 12, 2019. This work was supported in part by the Engineering and Physical Research Council under Award No. 169769

L. Forth, J. Khong, R.Moss and R.Speller are with the Department of Medical Physics and Biomedical Engineering, UCL, London, WC1E 6BT, UK, (emails: logan.forth.15@ucl.ac.uk, robert.moss@ucl.ac.uk, r.speller@ucl.ac.uk) M. Wang and K. Choy are with the Institute for Materials Discovery, UCL, London, WC1E 6BT, UK, (e-mails: mingqing.wang@ucl.ac.uk, k.choy@ucl.ac.uk)

I. Braddock and P. Sellin are with the Physics Department of The University of Surrey, Guildford, Surrey, GU2 7XH,, UK (emails: i.braddock@ surrey.ac.uk, p.sellin@surrey.ac.uk)
CdTe, Gadolinium Oxysulphide, these perovskite materials, particularly $\mathrm{CsPbBr}_{3}$ and $\mathrm{CsPb}_{3}$, offer a strong competitive edge from this higher internal efficiency for absorbing photons and converting them to extractable free charge carriers.

\section{AIMS}

The aim of this research project is to explore the capabilities of $\mathrm{CsPbBr}_{3} / \mathrm{I}_{3}$ as an X-ray detecting material and to consider the possible applications. A combination of device construction/testing and Monte Carlo simulation is needed to understand their full potential.

The detector designs being explored are of 4 types, shown in figure 1:

1) Flat planar electrodes covering the entire sample

2) Generic smaller shaped electrodes for looking at multiple selections of the samples

3) $2 \mathrm{D}$ pixel arrays of pitch $=50,100,200$ and 500 $\mu \mathrm{m}$

4) 1D pixel distribution of either even or varying pitch

Design 1 is of use for measuring the general properties of the samples after synthesis for overall bulk performance testing as the entire sample acts as an active area for detection. Design 2 is helpful for observing local variability within the samples themselves, if the response is constant across the entire sample.

The third design is using 2D pixel arrays, which are a well established means for spatially imaging an orthogonal beam of photons and the performance of such arrays in $\mathrm{CsPbr}_{3}$ must be tested, through which its application to the most common uses of X-rays in imaging can be determined.

The fourth design, of 1D pixels is more experimental, where the focus is to extract the photoexcited charge carriers to electrodes that are placed at set distances along the path of the incoming photons. The intention, as shown in figure 1b), is for photons to enter the detective area of the material from a lateral direction. The pixel distances are placed to correspond with depths within the perovskite that the photon is likely to be absorbed, which can function as an indirect measure of the energy of the photon, as higher energy photons will penetrate further before complete absorption. Another advantage to this design is that the only predetermined dimension is the attenuation length of the material, which is approximately 1 $\mathrm{mm}$ for most energies below $100 \mathrm{keV}$ as shown in fig 1c), leaving the thickness of material and width of electrodes to be optimised for efficiency or resolution in detector design. 


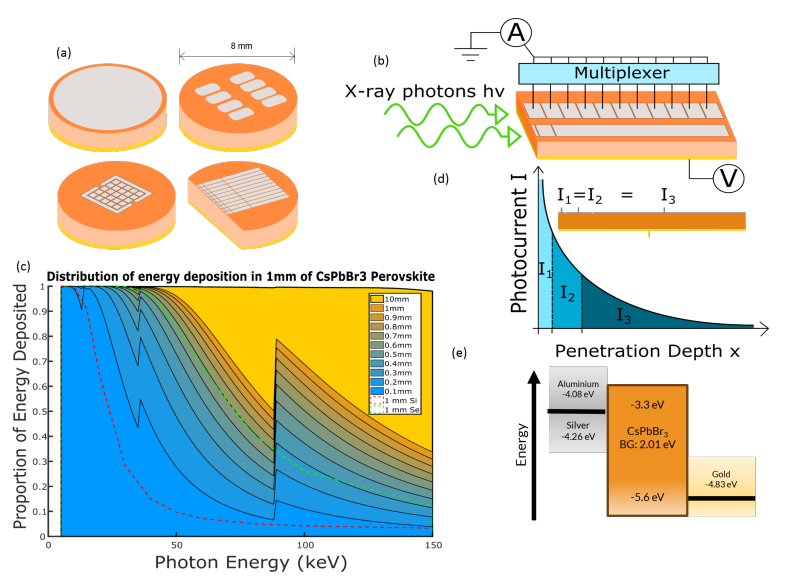

Figure 1.

a) A diagram of the 4 device designs

b) Diagram of the measurement method for device design 2

c) A graph from GEANT4 simulations comparing the attenuation efficiency of multiple thicknesses of $\mathrm{CsPbBr}_{3}$ for X-ray energies from 5 to $150 \mathrm{keV}$, with Silicon and Selenium included for comparison

d) A diagram of the theoretical distribution of photocurrent within a device of design two for a beam of monoenergetic X-rays

e) Band structure of the electrode interface between $\mathrm{Au}-\mathrm{CsPbBr}_{3}-\mathrm{Al} / \mathrm{Ag}$

\section{METHODS}

Figure 2 shows a diagram of the process for producing and functionalising devices. Initially the precursor reagents, $\mathrm{CsBr}$ and $\mathrm{PbBr}_{2}$ or $\mathrm{PbI}_{2}$, are mixed, at a 2:5 molar ratio, into a solution of Dimethylsulphoxide (DMSO). The solution is sealed but with air punctures and placed in a second sealed container with liquid Dicholoromethane (DCM). The crystals grow in solution over the course of 72 hours by the reactions of DMSO with DCM vapour. The crystals are removed and washed with Dimethylformamide (DMF) to remove the DMSO and dissolve impure crystal growth. After drying in a vacuum oven, the crystals are able to be processed into pellets, via two methods:

Method one is simply placing an adequate amount of Perovskite crystal $(300 \mathrm{mg})$ into a steel well, between two steel plates, and compressing with a hydraulic press at 784 MPa to form thin pellets of polycrystalline Perovskite.

Method is a modification of the methodology used in [2] two aims to produce single crystals of Perovskite. First, the sample is placed into a quartz tube, evacuated and sealed by glassblowing. The resultant ampoule is then heated in furnace oven up to $600{ }^{\circ} \mathrm{C}$, and slowly cooled by $6{ }^{\circ} \mathrm{C}$ per hour to form the crystal over 3 days. After the cooling, the ampoules are broken and the crystals are sliced into $12 \mathrm{~mm}$ thick pellets by wire saw.

After forming polycrystalline and monocrystalline Perovskite pellets, the crystals are functionalised in two ways. Contacts were deposited on the materials using thermal evaporation of silver or aluminium with a shadow mask to form 2D pixel arrays or 1D channel contacts and then attached to a gold substrate using silver paste. Designs for evaporation deposition include general surface contacts, $5 \times 5$ pixel contacts of sizes varying from $50-500 \mu \mathrm{m}$, and various lengths of separated channels. Contact with the devices was made

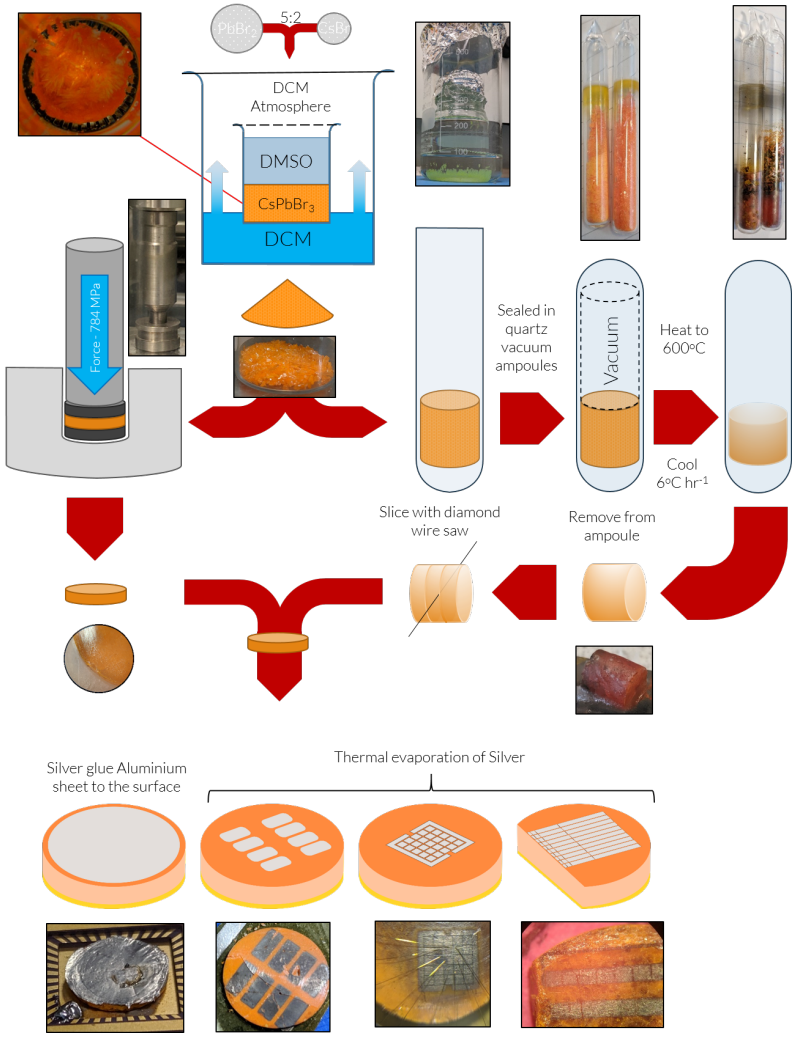

Figure 2. A flow chart diagram of the beginning to end production method for the Perovskite devices. Steps are accompanied by inset images from each stage of production and the finished products.

through soldering to large pads or gold wire bonding for the smaller pads such as the pixels and channels.

The devices were exposed to X-rays from a tungsten target $\mathrm{X}$-ray source operating at energies and currents ranging from $20-100 \mathrm{kVp}$ and $1-10 \mathrm{~mA}$ respectively. X-ray photocurrent was measured using a Keithley 6487 picoammeter, used in conjunction with a multiplexer for pixel and channel readout.

\section{RESUlTS AND Discussion}

Figure 3 shows results from tests carried out on a sample of the crushed powder Perovskite. The dark current, shown in the inset in fig 3 at the beginning of the exposure, is determined to be $8.2 \mathrm{pA}$, and a maximum response to X-rays is observed at $4.2 \mathrm{nA}$, an increase of current over 500 times more than the dark current. Photoluminescence performed on the samples revealed some presence of the stable crystal structure of $\mathrm{CsPbBr}_{3}$ while also large compositions of contaminants at the surface of the crystallites, including unreacted lead bromide and caesium bromide, caesium nitride and lead bromine oxide along with some alternative phases of $\mathrm{CsPbr}_{3}$.

Figure 4 shows an array of results from more preliminary tests carried out on samples of multiple manufacture methods. The most promising of these initial results is shown in 4a) where at maximum X-ray exposure a $\mathrm{I}_{\mathrm{ON}} / \mathrm{I}_{\mathrm{OFF}}$ ratio of 86.2 was observed, and 1.6 at minimum $\mathrm{X}$-ray exposure, with a dark current of $0.7 \mathrm{nA}$ at $5 \mathrm{~V}$ bias. This sample was produced from crushing the ground crystal powder together into a 10 $\mathrm{mm}$ pellet and deposition of silver contacts. Figure $4 \mathrm{~b}$ ) shows 


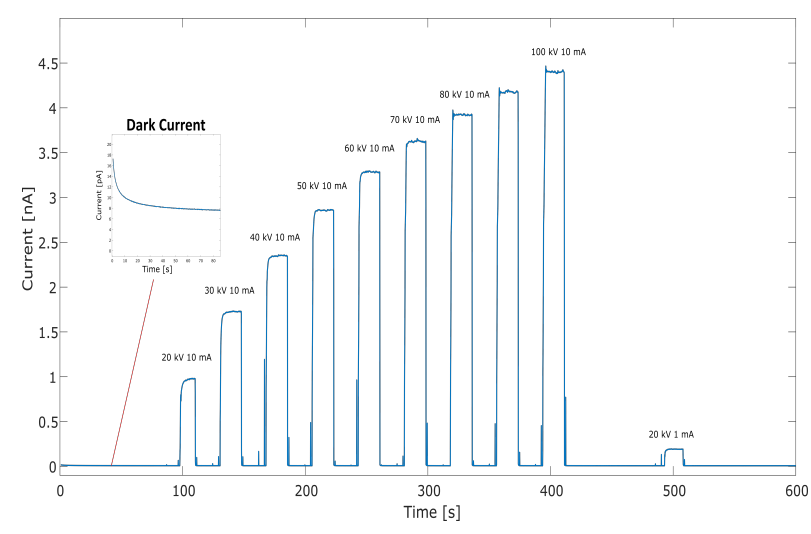

Figure 3. Response of a device to X-ray exposure from 20 to $100 \mathrm{kVp}$ and $10 \mathrm{~mA}$, with one exposure of $20 \mathrm{kV}$ and $1 \mathrm{~mA}$, inset with the dark current obtained in the first 80 seconds of operation and an image of the device under test

the responses of a single crystal based device. This single crystal was produced by furnace heating and gradual cooling to form the lattice structure. The dark current here is much greater, but this device was not set up with any charge blocking layers, so this could easily be reduced in future. 4c) shows the response of a $1 \mathrm{~mm}$ thick crushed powder sample, through a bias sweep. The response here is only about 4 nA. Figure $4 d$ ) shows a much greater response from the same sample after being soldered into the circuit.

Figure 5, shows results from more recent devices, with a variety of performance and reliability, along with insets of the devices under test. Fig 5a) shows the response of a single pixel in the centre of the $5 \times 5$ pixel array. The dark current lies around $10 \mathrm{pA}$ and photoresponse ranges from 0.8 up to $4 \mathrm{nA}$. A similar device response is also seen in fig 5b) with a dark current of $8 \mathrm{pA}$ and a higher response of $4.4 \mathrm{nA}$. These two
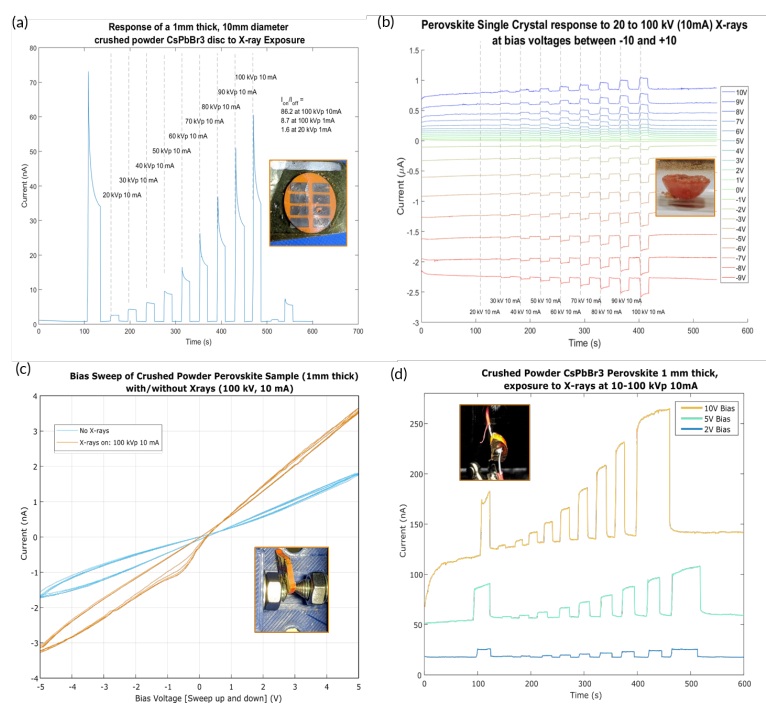

Figure 4. Direct X-ray exposure response of $3 \mathrm{CsPbBr}_{3}$ samples with inset images of each sample

a) $500 \mu \mathrm{m}$ thick sample featuring a maximum $\mathrm{I}_{\mathrm{ON}} / \mathrm{I}_{\mathrm{OFF}}$ ratio of 86

b) $5 \mathrm{~mm}$ single crystal biased at -9 to $+10 \mathrm{~V}$, produced from a furnace melt

c) Bias voltage sweep from -5 to $+5 \mathrm{~V}$ for a $1 \mathrm{~mm}$ thick sample

d) $1 \mathrm{~mm}$ sample from c) soldered for a more direct connection
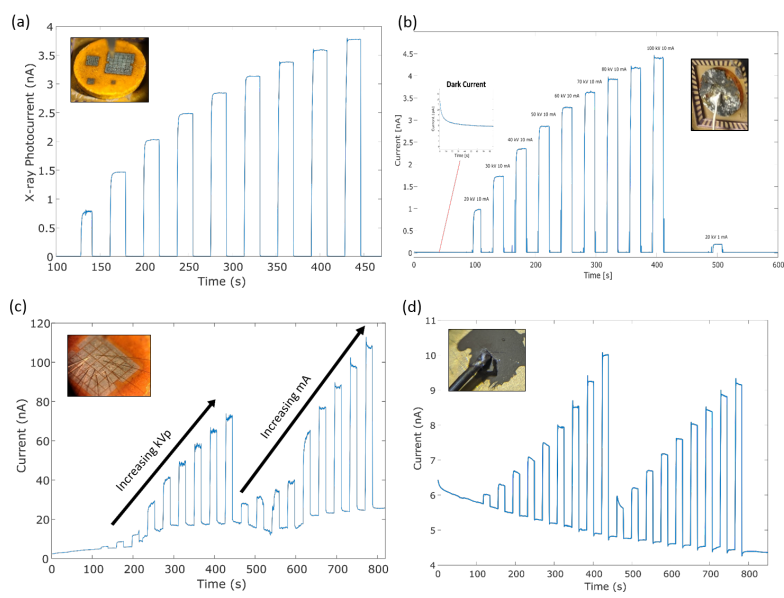

Figure 5. Responses of various devices to X-ray exposure varying from 20 $100 \mathrm{kVp}$ and $110 \mathrm{~mA}$ and photoluminescence emission measurements of crystal samples

a) A single $500 \mu \mathrm{m}$ silver contact pixel on a $1.1 \mathrm{~mm}$ pressed pellet photocurrent

b) A $1 \mathrm{~mm}$ pressed pellet with an Aluminium sheet

c) The central pixel in an array of $3 \times 3$ connected pixels

d) Small single crystallite shard connected with silver glue and solder

devices exhibit incredibly high dark current to photoresponse ratios of around 450 . Figure $5 \mathrm{c}$ ) shows the response of the central pixel in a grid of $500 \mu \mathrm{m}$ wide pixels. Here the dark current is much greater than the previous two devices and unstable from $3 \mathrm{nA}$ up to $24 \mathrm{nA}$. However, the total response of the device is much greater also, up to $111 \mathrm{nA}$. In fig $5 \mathrm{~d}$ ) the response of a small ( $\sim 600 \mu \mathrm{m}$ across) single crystal shard is shown. The dark current here is a large proportion of the maximum response, with a ratio as low as 1.05 to 2 , and also decreasing with time along an inverse square law.
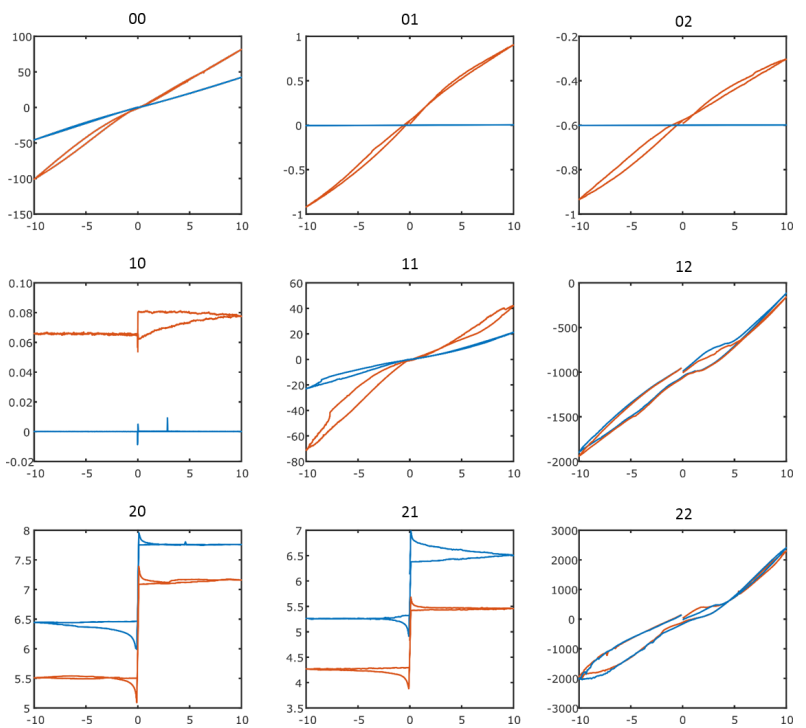

Figure 6. A $3 \times 3$ plot of IV-Bias curves measured for the each of the 500 $\mu \mathrm{m}$ pixels of the $3 \times 3$ grid in fig $5 \mathrm{c}$, between -10 and $10 \mathrm{~V}$, where the current measured in nA. Shown in blue is the pixel under dark exposure with no $\mathrm{X}$ rays or light present, and in orange is the pixels performance under exposure to $100 \mathrm{kV} 10 \mathrm{~mA} \mathrm{X}$-rays. The pixels are labelled by their row and column index from 0 to 2 . 

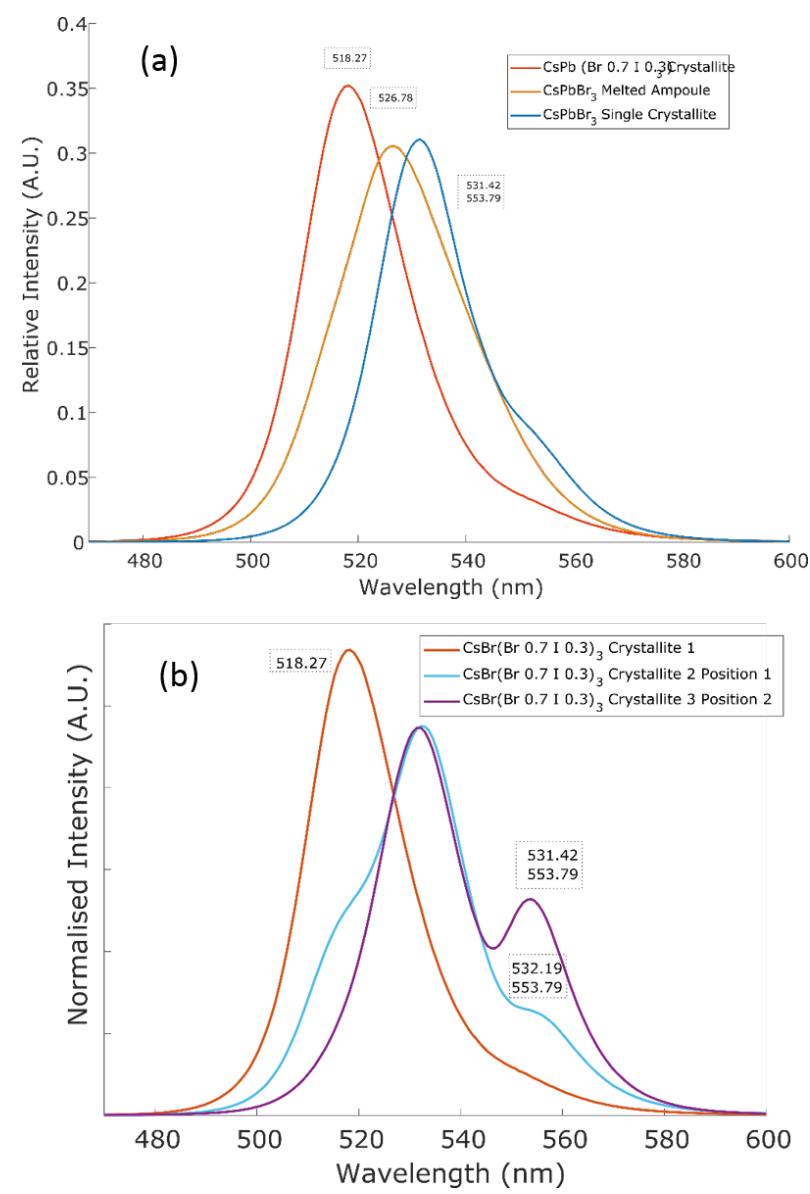

Figure 7.

a) Photoluminescence peaks of 3 separate samples of $\mathrm{CsPbBr}_{3} / \mathrm{I}_{3}$, highlighting the bandgaps

b) Photoluminescence peaks of $\mathrm{CsPb}\left(\mathrm{Br}_{0.7} \mathrm{I}_{0.3}\right)_{3}$, highlighting the bandgaps variation within multiple positions on the same sample

Figure 6 features a $3 \times 3$ grid of voltage bias curves for all 9 central pixels of the same device in fig $5 \mathrm{c}$ ), under standard dark conditions and also under $100 \mathrm{kVp} 10 \mathrm{~mA} \mathrm{X}$-ray exposure. Each pixel appears to function distinctly. For example pixel 01 and 02 appear to have a similar level of response to the pixel in $5 \mathrm{a}$ ), while pixel 00 and 11 respond more similarly to the pixel in 4c). Pixels 12 and 22 have a much lower resistance to the bias and so have currents of greater than $1 \mu \mathrm{A}$. Pixels 10, 20 and 21 appear to be disconnected or dysfunctional, however there is still difference in the current from the X-ray response.

Figure 7 shows photoluminescence emission measurements of various samples aiming to distinguish the bandgaps and compound distribution of the crystals. Figure 7a) highlights the shift upwards of the bandgap observed between different forms of the Perovskite sample, from a crystal fragment prior to processing, to a melt produced single crystal of $\mathrm{CsPbBr}_{3}$ to a crystal fragment of $\mathrm{CsPb}\left(\mathrm{Br}_{0.7} \mathrm{I}_{0.3}\right)_{3}$. Fig 7b) shows that within a single sample of the Iodine doped crystal a variety of bandgap intensities can be observed from different positions on the sample, highlighting multigrain mixes of compounds and sub crystal clumping.

Figure 8 features an $\mathrm{XY}$ scan of a collimated X-ray beam using the small crystal shard sample from fig $5 \mathrm{~d}$ ). The scan

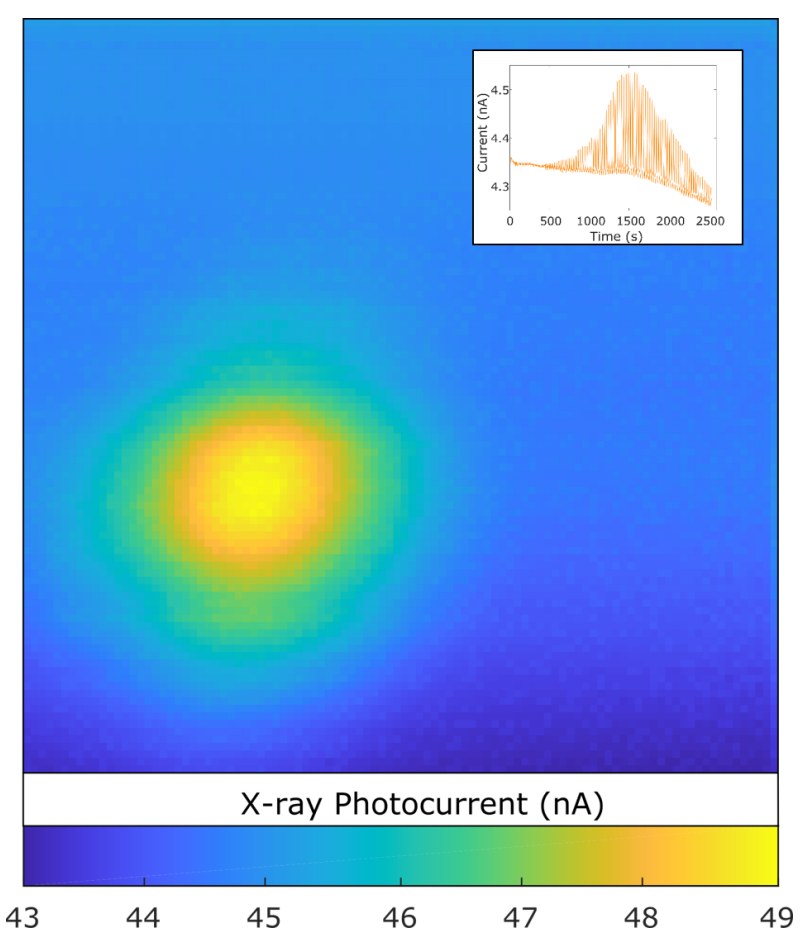

Figure 8. A $20 \times 20 \mathrm{~mm}$ scan of an X-ray beam with the device from fig 4 $\mathrm{d}$, with an inset showing the photocurrent over time

area is $20 \times 20 \mathrm{~mm}$, taken at $100 \times 100$ points, essentially using the shard as a pixel and forming an image of the X-ray beam. The X-ray beam was collimated to $6.4 \mathrm{~mm}$ diameter (at the sample) pencil beam and the responsive region of the image corresponds to that width, within the error of the lead collimation window. The inset shows the response during the measurement of the image, within which time the dark current decreases sporadically, but a functional image is still observed, despite only a $20 \%$ increase in current over the dark current. This is the first usage of the Perovskite crystals in this study for building an image and can potentially be used in future using subjects to test the imaging capabilities of these devices.

\section{CONCLusions}

These devices have shown a variety of responses and reliability under X-ray illumination. It was possible to observe photocurrents generated of up to $100 \mathrm{nA}$, with a variety of sensitivities relative to the dark current. Device results with the most impressive photoresponse to dark current ratios also have some of the lowest total photocurrent response, while devices with high response also exhibit much larger dark currents. However, even devices with the lowest performance ratio were useful in detecting X-ray photocurrents. Results from the channelled devices would prove very interesting for fully realising the application of this new material. The results overall indicate that these device designs are promising though require optimisation for further application. The material production employed a low cost method and made efficient use of the materials required, especially through the pressed powder production, which may enable low cost sensitive direct X-ray detector fabrication in future. 


\section{REFERENCES}

[1] Y. He, W. Ke, G. C. B. Alexander, K. M. McCall, D. G. Chica, Z. Liu, I. Hadar, C. C. Stoumpos, B. W. Wessels, and M. G. Kanatzidis, "Resolving the Energy of -Ray Photons with MAPbI3 Single Crystals," ACS Photonics, vol. 5, no. 10, pp. 4132-4138, Oct. 2018. [Online]. Available: https://doi.org/10.1021/acsphotonics.8b00873

[2] Y. He, L. Matei, H. J. Jung, K. M. McCall, M. Chen, C. C. Stoumpos, Z. Liu, J. A. Peters, D. Y. Chung, B. W. Wessels, M. R. Wasielewski, V. P. Dravid, A. Burger, and M. G. Kanatzidis, "High spectral resolution of gamma-rays at room temperature by perovskite $\mathrm{CsPbBr} 3$ single crystals," Nature Communications, vol. 9, no. 1, p. 1609, Apr. 2018. [Online]. Available: https://www.nature.com/articles/s41467-018-04073-3

[3] R. Wang, M. Mujahid, Y. Duan, Z.-K. Wang, J. Xue, and Y. Yang, "A Review of Perovskites Solar Cell Stability," Advanced Functional Materials, vol. 0, no. 0, p. 1808843, Jan. 2019. [Online]. Available: https://onlinelibrary.wiley.com/doi/abs/10.1002/adfm.201808843 